\title{
A New Distributed Type-2 Fuzzy Logic Method for Efficient Data Science Models of Medical Informatics
}

\author{
Fatéma Zahra Benchara $(D)$ and Mohamed Youssfi \\ Department of Computer Science, Laboratory SSDIA, ENSET Mohammedia, Hassan II University of Casablanca, \\ Casablanca, Morocco \\ Correspondence should be addressed to Fatéma Zahra Benchara; benchara.fatemazahra@gmail.com
}

Received 5 January 2020; Revised 24 April 2020; Accepted 16 June 2020; Published 25 August 2020

Academic Editor: Antonin Dvorák

Copyright (C) 2020 Fatéma Zahra Benchara and Mohamed Youssfi. This is an open access article distributed under the Creative Commons Attribution License, which permits unrestricted use, distribution, and reproduction in any medium, provided the original work is properly cited.

\begin{abstract}
The paper aims to propose a distributed method for machine learning models and its application for medical data analysis. The great challenge in the medicine field is to provide a scalable image processing model, which integrates the computing processing requirements and computing-aided medical decision making. The proposed Fuzzy logic method is based on a distributed approach of type-2 Fuzzy logic algorithm and merges the HPC (High Performance Computing) and cognitive aspect on one model. Accordingly, the method is assigned to be implemented on big data analysis and data science prediction models for healthcare applications. The paper focuses on the proposed distributed Type-2 Fuzzy Logic (DT2FL) method and its application for MRI data analysis under a massively parallel and distributed virtual mobile agent architecture. Indeed, the paper presents some experimental results which highlight the accuracy and efficiency of the proposed method.
\end{abstract}

\section{Introduction}

Today, computer science technologies apply artificial intelligence and data science models in order to design new intelligent applications, such as fraud detection and recommendation engine. These applications have to deal with big data sets, which need to be processed for extracting meaningful information and predict unknown patterns. To do so, they introduce the use of machine learning models based on complex algorithms such as classification and clustering algorithms.

Consider the great number of unstructured datasets and the complex algorithms that these applications have to deal with. Their efficiency relies on their capability to manage these challenges. For example, running a medical application for image analysis requires high processing power and a scalable clustering method. Clustering algorithms which are widely applied in the medicine field have been used by many researchers for MRI image classification. As an illustration, they proposed a parallel Fuzzy c-means method for image segmentation analysis in [1] and for clustering large data sets on a parallel SPMD architecture using MPI tools in [2]. For the type-2 Fuzzy, some parallel implementation are proposed such as in [3] over FPGA technology. The question of how distributed clustering methods can improve the big data analysis and provide an effective tool for decision making models then arises. Consider the considerable requirements of medical big data analysis and decision making models based on clustering methods. The paper focused on presenting a cooperative machine learning model based on the distributed type-2 Fuzzy method that combines computational processing requirements and the cognitive aspect. This paper is organized as follows:

We will describe the machine learning model-based mobile agents and their main components, which are a team leader agent and AVPEs agents (Section 3)

We will focus on presenting the proposed distributed DT2FL method (Section 4) and demonstrating its promising advantages through medical image analysis application (Section 5) 


\section{Background}

To highlight the aim of this paper, we start with a brief overview of machine learning models [4,5] and their ability to perform prediction, pattern recognition, and decision making. These models provide the computers by the teaching behavior to learn from datasets and lead humans to convey their field expertise to them in order to design and implement smart systems, which are not explicitly programmed. These, later, are able to collaborate with humans and bring relevant solutions. For example, in the banking sector, the machine learning model is implemented in order to provide a fraud-detection system able to detect the fraudulent transactions. It is also implemented in social media for face recognition and in commerce for product recommendation to the customers. Therefore, there are three types of machine learning methods, where each one deals with a specific kind of problems.

Supervised learning: the algorithms of this type learn from training datasets in order to predict outcomes, where the output results are given in the training process

Unsupervised learning: the algorithms of this type find patterns without using training datasets. Examples of these algorithms are clustering and anomaly detection

Reinforcement learning: the algorithms of this type make decisions according to their past experience

The clustering algorithms are based on complex computing tasks and large datasets. They have a variety of applications such as image segmentation, medical imaging, and anomaly detection. Thus, it seems that these methods play a great role in designing and implementing effective machine learning models. This means that their performance depends on the clustering's algorithm scalability. The distributed clustering method is a new paradigm that allows performing tasks in distributed nodes. Assume that the task Tneeds to be performed on large datasets $D$. The task $T$ will be split into two global tasks, one executed by the host node and the second executed by all the slave nodes in the model. Furthermore, the data $D$ will also be split into (me $\times$ ne) elementary data $D j\{j=1, \ldots,($ me $\times$ ne $)\}$ and distributed to the node, by the way that they perform collaborative clustering tasks.

The multiagent system (MAS) [6] is a distributed technology system composed of a set of distributed agents which live and cooperate between each other using their intelligence and skills in different environments in order to overcome complex challenges. For example, in [7], the authors proposed a platform to facilitate the provision of home-care services based on an agent three-layered architecture. In [8], the authors presented the improvement of the time efficiency of a medical reasoning system based on a multiagent architecture. Thus, how can the distributed clustering paradigm and mobile agents be both the promising solution for scalable clustering methods and machine learning models?

\section{Autonomous Fuzzy Logic Model for Data Analysis}

The distributed type-2 Fuzzy logic method is a new efficient and accurate machine learning model (Figure 1) for performing medical applications in a distributed system. The proposed method is based on cooperative mobile agents as Agent Virtual Processing Elements (AVPEs) in order to perform complex computational tasks and process intensive datasets over a distributed virtual architecture. Clustering techniques are one of the unsupervised machine learning methods which need intensive computational power. The proposed method provides the computational capabilities for these requirements. Although, the cognitive aspect of the computational team enhances the method features in order to provide accurate medical decision making. For example, in order to execute an MRI image segmentation-based type- 2 Fuzzy clustering method, when the application is deployed, the cooperative DT2FL team works will be created. Its main components are the team leader agent and AVPEs (Agent Virtual Processing Elements) agents. The team leader agent is initialized by the input dataset, which is partitioned into (me $\times$ ne) elementary images and deploys $\mathrm{NA}=($ me $\times$ ne $)$ AVPEs agents. Each AVPE agent encapsulates its dataset and achieves tasks and sends the results to their team leader agent. At the end, the team leader agent gets the final results, which are used for accurate medical diagnosis. This is performed by the team leader agent under an embedded model, which enables autonomous detection of medical diseases and healthcare prediction (Figure 1).

\section{Distributed Type-2 Fuzzy Logic Method}

4.1. Standard Method. The Type-2 Fuzzy algorithm [9] is an extended version of Fuzzy c-means algorithm, and which is based on the method concepts for data clustering to $\mathrm{c}$ clusters. Beside, the Type-2 Fuzzy method integrates a new membership matrix aij assigned to the membership matrix. The method grants the convergence of the class centers to accurate values in the presence of noise compared to the Fuzzy c-means, and which minimize the objective function J given by the following equation:

$$
J=\sum_{i=1}^{c} J_{i}=\sum_{i=1}^{c} \sum_{j=1}^{n} a_{i j}^{m} d^{2}\left(v_{i}, x_{j}\right) .
$$

In each iteration, the new class centers and the membership matrices are computed, respectively, by the following equations:

$$
\begin{gathered}
V_{i}=\frac{\sum_{j=1}^{N} a_{i j}^{m} x_{j}}{\sum_{j=1}^{N} a_{i j}^{m}}, \\
a_{i j}=u_{i j}-\frac{\left(1-u_{i j}\right)}{2},
\end{gathered}
$$




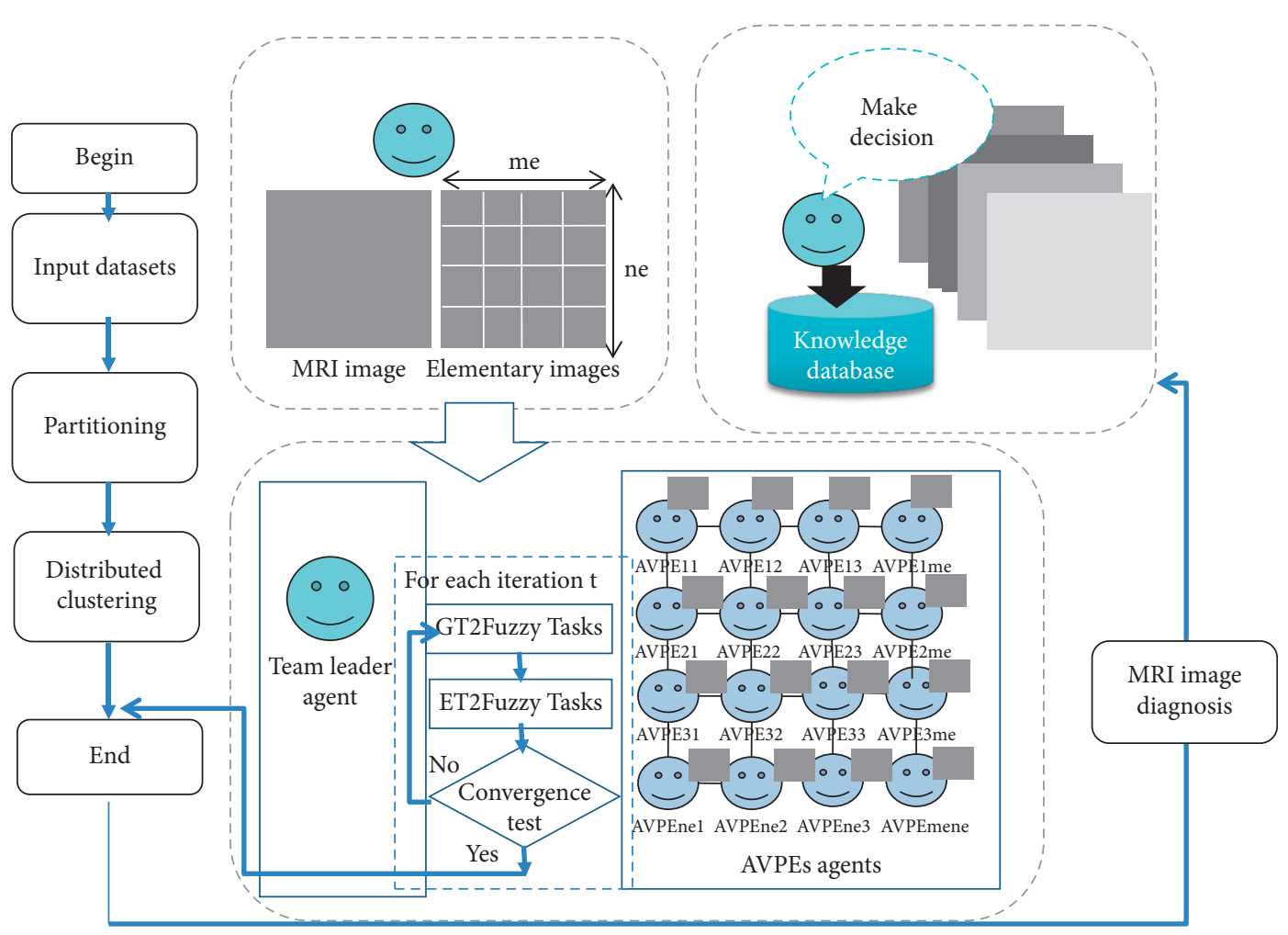

FIgURE 1: Autonomous clustering model based on a mobile agent.

$$
u_{i j}=\frac{1}{\sum_{k=1}^{c}\left(d_{i j} / d_{k j}\right)^{2 /(m-1)}} .
$$

The clustering of datasets based on standard Type- 2 Fuzzy algorithm (Figure 2) is achieved according to the following algorithm's steps:

\section{Step 1: Initialization}

(i) Initialize the fuzzification parameter.

(ii) Choose the number of clusters $C$.

(iii) Initialization of the clusters centers $V_{i}^{(0)}(i=0, \ldots, c)$ and the threshold $E_{\mathrm{th}}$.

For each iteration $t$

\section{\{}

Step 2: Determination of the membership matrices

(i) Determine the membership matrix $u_{i j}$ by (4).

(ii) Determine the membership matrix $a_{i j}$ by (3).

Step 3: Compute the objective function $J$ by (1).

Step 4: Test of convergence

if $\left(\left|J_{t}-J_{t-1}\right|<E_{\mathrm{th}}\right)$

the algorithm converges (i.e. go to step 6). else

go to step 5 .

Step 5: Calculate the new class centers $V_{i}^{(t)}$ by (2), and repeat the loop from step 2 .
Step 6: End.

\}

4.2. Distributed Method. This section is investigated to present the proposed distributed type-2 Fuzzy algorithm (DT2FL) and its implementation in cooperative distributed virtual architecture based on mobiles agents. The input dataset of the machine learning model is loaded in a virtual architecture of size $(\mathrm{me} \times \mathrm{ne})$. In the model, each agent virtual processing element $(\operatorname{AVPE}(\mathrm{g}))$ handles its assigned elementary dataset ed(e) and performs the different algorithm's steps. The computational algorithm's steps are achieved by the team leader agent in cooperation with the AVPEs agents in parallel, within distributed grid computing. The proposed DT2FL algorithm involves the 3 steps: initialization, grid construction, and distributed type2 Fuzzy clustering, which are summarized in (Figure 3) and detailed as follows:

4.2.1. Step 1: Initialization. In this step, the team leader agent is initialized by the required algorithm's inputs:

Dataset

Number of clusters c

The value of column ne and line me needed for creating the AVPE grid of size (me, ne)

4.2.2. Step 2: Grid Construction. In this step, the team leader agent starts by partitioning the input dataset $D$ into a set of 


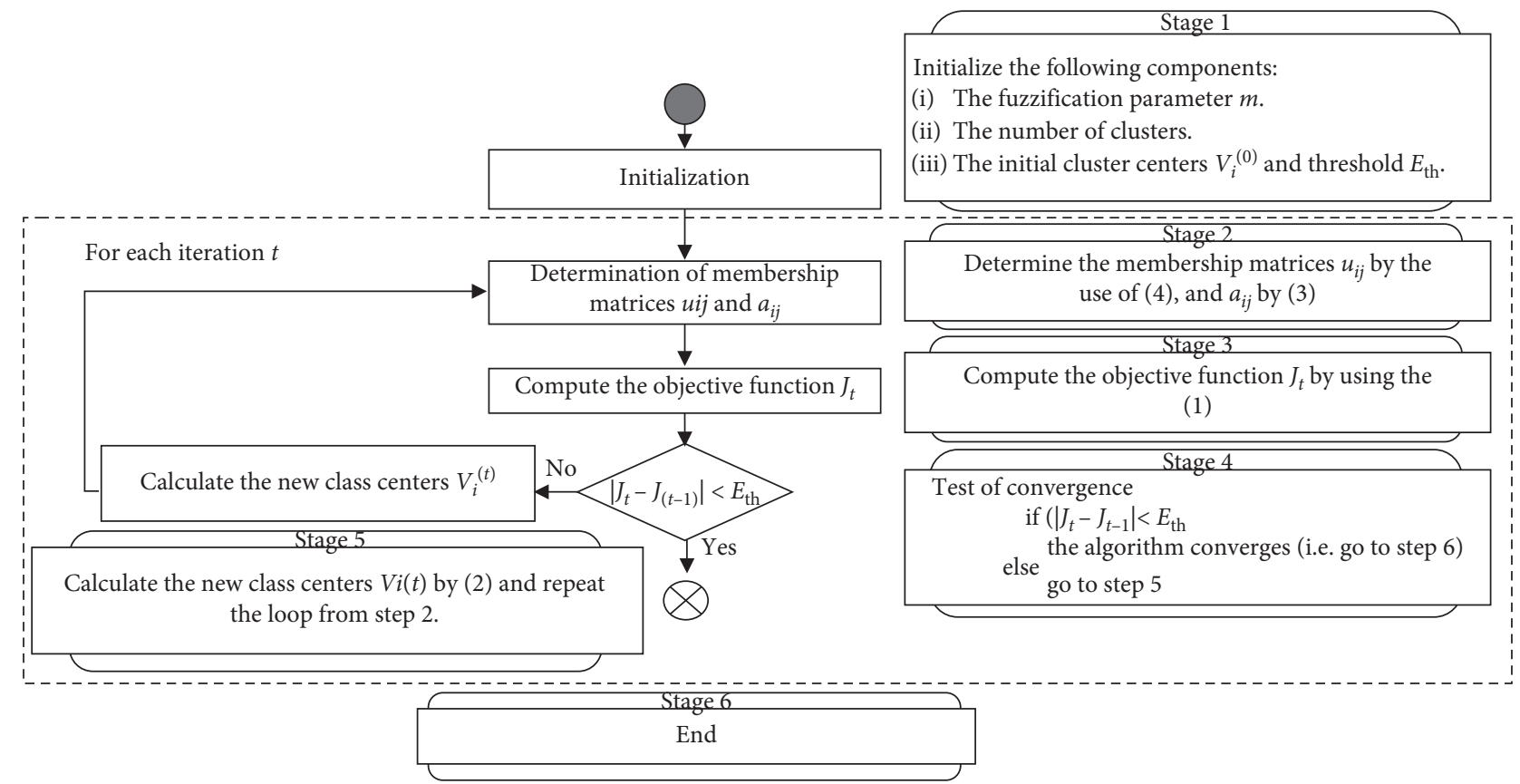

Figure 2: Standard type-2 Fuzzy logic algorithm's step.

$(\mathrm{me} \times \mathrm{ne})$ elementary dataset. Then, it deploys $\mathrm{NA}=(\mathrm{me} \times \mathrm{ne})$ AVPE agents, which are initialized by the elementary dataset (Figure 4), one per $\operatorname{AVPE}(\mathrm{g})$. When it is performed, each $\operatorname{AVPE}(\mathrm{g})$ moves to its appropriate distributed node, where it is supposed to perform its tasks.

4.2.3. Step 3: Distributed Type-2 Fuzzy Clustering. In this step, the team leader agent chooses the initial class centers $V_{i}^{(0)}(i=1, \ldots, \mathrm{c})$ and the threshold $E_{\mathrm{th}}$, while each $\operatorname{AVPE}(\mathrm{g})$ encapsulates the tasks and loads its elementary dataset $e$.

For each iteration $t$,

\{

1: the team leader agent sends the class centers to all the AVPEs.

2: each agent $\operatorname{AVPE}(\mathrm{g})$ gets the class centers from its message and performs the distributed elementary clustering task. Besides, each one determinates the membership matrix $u_{i j}$, and $a_{i j}$, and sends the results DER1, DER2, and DER3 to the team leader agent. These results consist of the following components:

$$
\begin{aligned}
& \operatorname{DER} 1(g, i)=\sum_{j=1}^{d i} a_{i j}^{m} x_{j}, \\
& \operatorname{DER} 2(g, i)=\sum_{j=1}^{d i} a_{i j}^{m}, \\
& \operatorname{DER} 3(g)=\sum_{j=1}^{d i} \sum_{i=1}^{c} a_{i j}^{m} d^{2}\left(v_{i}, x_{j}\right),
\end{aligned}
$$

where $\operatorname{DER} 1(g, i)$ contains the results of the sum of $\left(A^{m} \times e\right)$ computed for each class center $i$. DER2 $(g, i)$ contains the results of the sum of $\left(A^{m}\right)$ computed for each class center $i$. DER3(g) contains the results of sum of $\left(A^{m} \times\right.$ distance $\left.^{2}\right)$ computed for all class centers. $d i$ is the number of data of the elementary dataset $e$ of the AVPE(g).

3: the team leader agent performs the global clustering task, within the three following subtasks:

Assembling the elementary results: the team leader agent receives the AVPEs results (DER1, DER2, and DER3) and computes the global results GDER1, GDER2, and GDER3:

$$
\begin{aligned}
& \operatorname{GDER} 1(i)=\sum_{g=1}^{N A} \operatorname{DER} 1(g, i), \\
& \operatorname{GDER} 2(i)=\sum_{g=1}^{N A} \operatorname{DER} 2(g, i), \\
& \operatorname{GDER} 3(i)=\sum_{g=1}^{\text {NA }} \operatorname{DER} 3(g),
\end{aligned}
$$

where GDER1( $i)$ is the global value of $\operatorname{DER} 1(g, i)$ of all the AVPEs of the model. GDER2( $i)$ is the global value of $\operatorname{DER} 2(g, i)$ of all the AVPEs of the model. GDER3 $(i)$ is the global value of DER3 $(g, i)$ of all the AVPEs of the model.

Computing the class centers: the team leader agent computes the new class centers $V_{i}$ by getting the computed global values GDER1(i) and GDER2(i) by (11)

$$
V_{i}=\frac{\operatorname{GDER} 1(i)}{\operatorname{GDER} 2(i)}, \quad i=1, \ldots, c .
$$




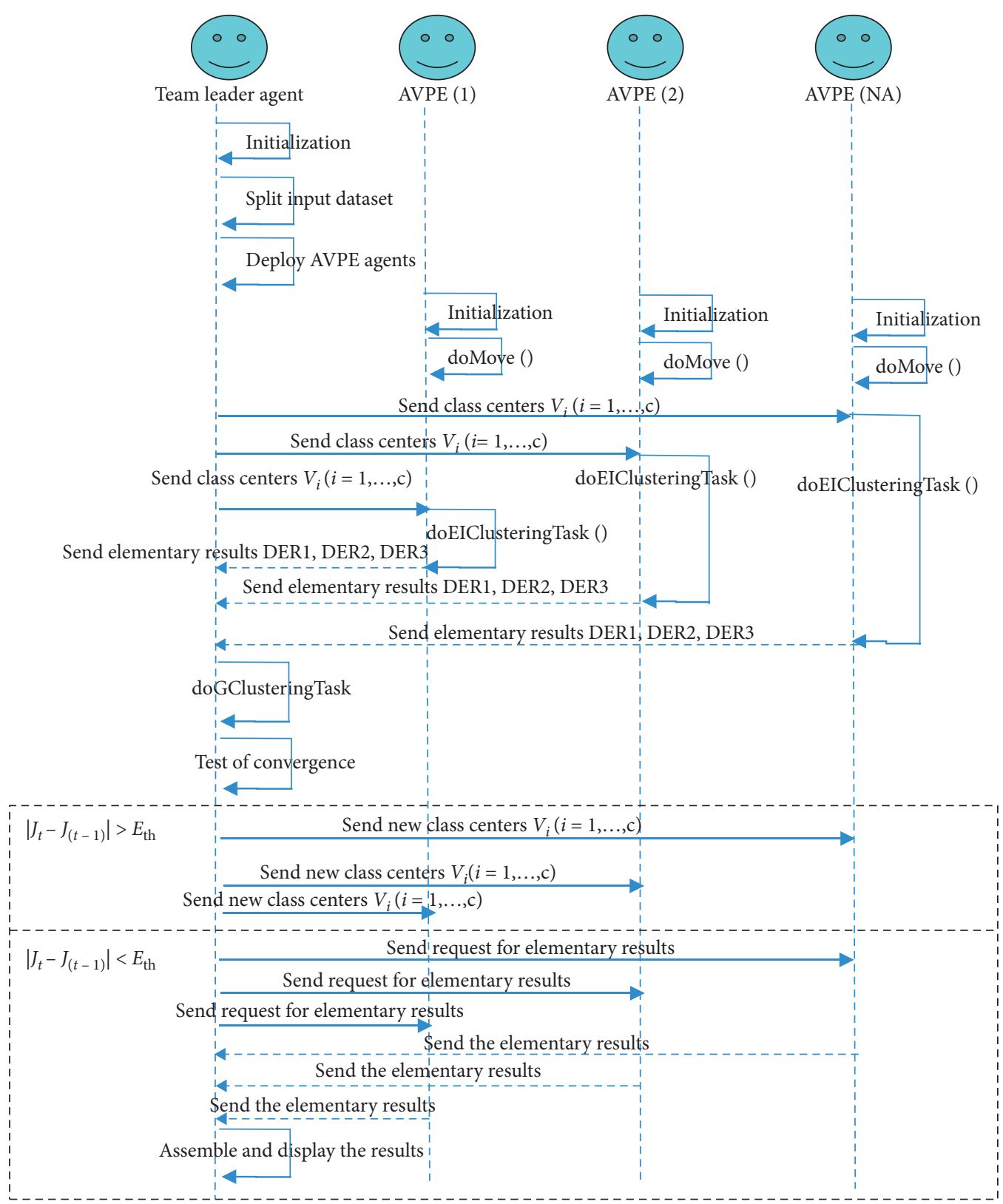

Figure 3: Distributed type-2 Fuzzy logic method based on mobile agent team work.

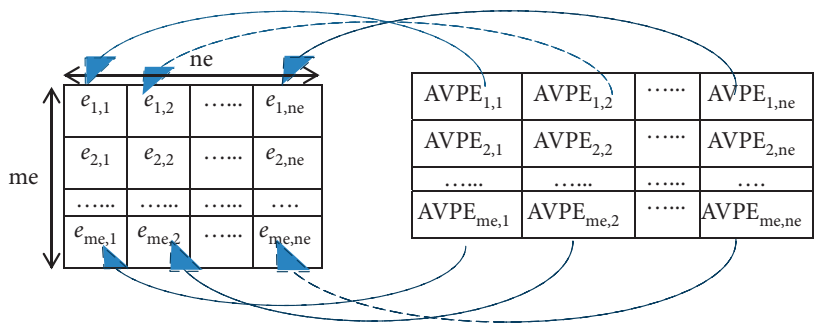

Figure 4: Overview of AVPEs agents data initialization. 
Computing the objective function $J_{t}$ : the team leader agent computes the objective function by getting the computed global values GDER1(i) by (12)

$$
J_{t}=\sum_{i=1}^{c} \operatorname{GDER} 3(i) .
$$

\section{Implementation and Results}

The machine learning model-based distributed type-2 Fuzzy logic is implemented in a distributed computing virtual machine for running an MRI medical image analysis application. The input dataset (MRI image) is encapsulated on the team leader agent and partitioned into elementary images. After creating the grid computing, the team leader, in cooperation with the AVPEs agents, will perform distributed method tasks and provide the results, Figure 5. The effectiveness of this method is presented under the dynamic convergence and the speedup of the algorithm.

5.1. Dynamic Convergence. The proposed DT2FL method is executed with different initial class centers initializations. The convergence results are presented as follows:

$(\mathrm{c} 1, \mathrm{c} 2, \mathrm{c3}, \mathrm{c4}, \mathrm{c5})=(1.5,2.2,3.8,5.2,8.6)$ : From Table 1 and Figure 6, it is clear that the dynamic convergence of the algorithm to the final class centers $(\mathrm{c} 1, \mathrm{c} 2, \mathrm{c3}, \mathrm{c4}, \mathrm{c5})=(27$. $711,82.848,89.817,103.896,94.371)$. The convergence is achieved after 23 iterations.

$(\mathrm{c} 1, \mathrm{c} 2, \mathrm{c3}, \mathrm{c4}, \mathrm{c5})=(140.5,149.5,150.5,220.2,250.5)$ : the algorithm converges to the final class centers (c1, c2, $\mathrm{c} 3, \mathrm{c} 4, \mathrm{c} 5)=(27.703,82.955,89.623,103.884,94.263)$ after 49 iterations as illustrated in Table 1 and Figure 7.

5.2. Clustering Time. In order to illustrate the effectiveness of the proposed model for data analysis, a comparative study is performed between the proposed DT2FL method and DFCM method in [10], by using the clustering time variation as metric according to the AVPEs agents.

From Table 2, we see clearly that the clustering time of the DT2FL method using one agent corresponds to the sequential method. Indeed, we see clearly from Figure 8 that for both methods, the clustering time is saved and achieves minimum values from 16 AVPE agents.

5.3. Speedup. The speedup of distributed methods compared to sequential methods is shown in Figure 9. Indeed, we see clearly that the speedup reaches interesting values compared to the sequential method which exceeded 4 by using 16 AVPE agents for two methods.

\section{Related Work}

The main focus of this work is to propose a distributed method for big data analysis and medical image diagnosis.
This method used the mobile agent-based model and a distributed approach of the type-2 Fuzzy logic algorithm.

The type-2 Fuzzy logic method theory and applications $[11,12]$ provide the important features of this method and its future directions for modeling complex systems based on data uncertainty. It was proposed by Zadeh [13], and it is one of the methods that allow modeling human perception effectively [13] and where modeling uncertainty is enhanced compared to Fuzzy type-1 [14]. This method has had great interests in several inspiring works in the literature at different domains. For example, in the medical domain, this method is implemented [15] to develop an automated tumor detection system. It is also widely investigated for medical image segmentation [16] and data classification applications [17] as a combined method with wavelets. The method has taken place as well as in image processing [18], and pattern recognition fields [19]. The type-2 Fuzzy method has a great interest in medical applications [20] for decision making [21], where the method is embedded with the neural network model. To illustrate, the authors in [22] proposed a hybrid model for 2-lead cardiac arrhythmia classification based on neural networks and type- 1 and type-2 Fuzzy systems. Thus, the Type- 2 Fuzzy drives interesting works in medical, and several other fields, such as in [23-33].

In [27], the authors mention the great role of the cognitive agent's ability to handle complex tasks. They formulate, implement, and evaluate a cognitive agent, which combines learning by, examples, with machine learning. The Fuzzy logic-based agent approach is also the aim of interest of different works. In [28], the authors proposed multiagent model predictive control including Fuzzy negotiation, which is implemented in a JAVA-based platform. It is also investigated [29] for designing and implementing an intelligent method for information extraction when information is abundant, vague, or imprecise. Furthermore, the multiagent approach is investigated for solving problems in traffic fields, [30] and for decision making of maintenance [31] in real time, and for effective coordination scheme [32] based on Type-2 Fuzzy decision making. The combination of the two aspects, the Fuzzy logic and multiagent approach, provides promising solutions which lead to extension the standard type-2 Fuzzy logic algorithm to a new distributed one based on the multiagent architecture. Namely, in [33] the authors proposed a multiagent architecture for the design of the hierarchical interval type- 2 beta Fuzzy system.

Thus, these works' foundations are developed in the following way. The proposed distributed type-2 Fuzzy logic in this paper is based on the multiagent approach and implements the given properties:

Scalable medical MRI image analysis method, which is based on the distributed approach of type-2 Fuzzy logic algorithm, within mobile agents: this method grants fast distributed data analysis, within its computational type-2 Fuzzy clustering model.

Accurate machine learning model-based type-2 Fuzzy logic for medical application: this method allows deep data analysis, within its embedded cognitive decision making model. 


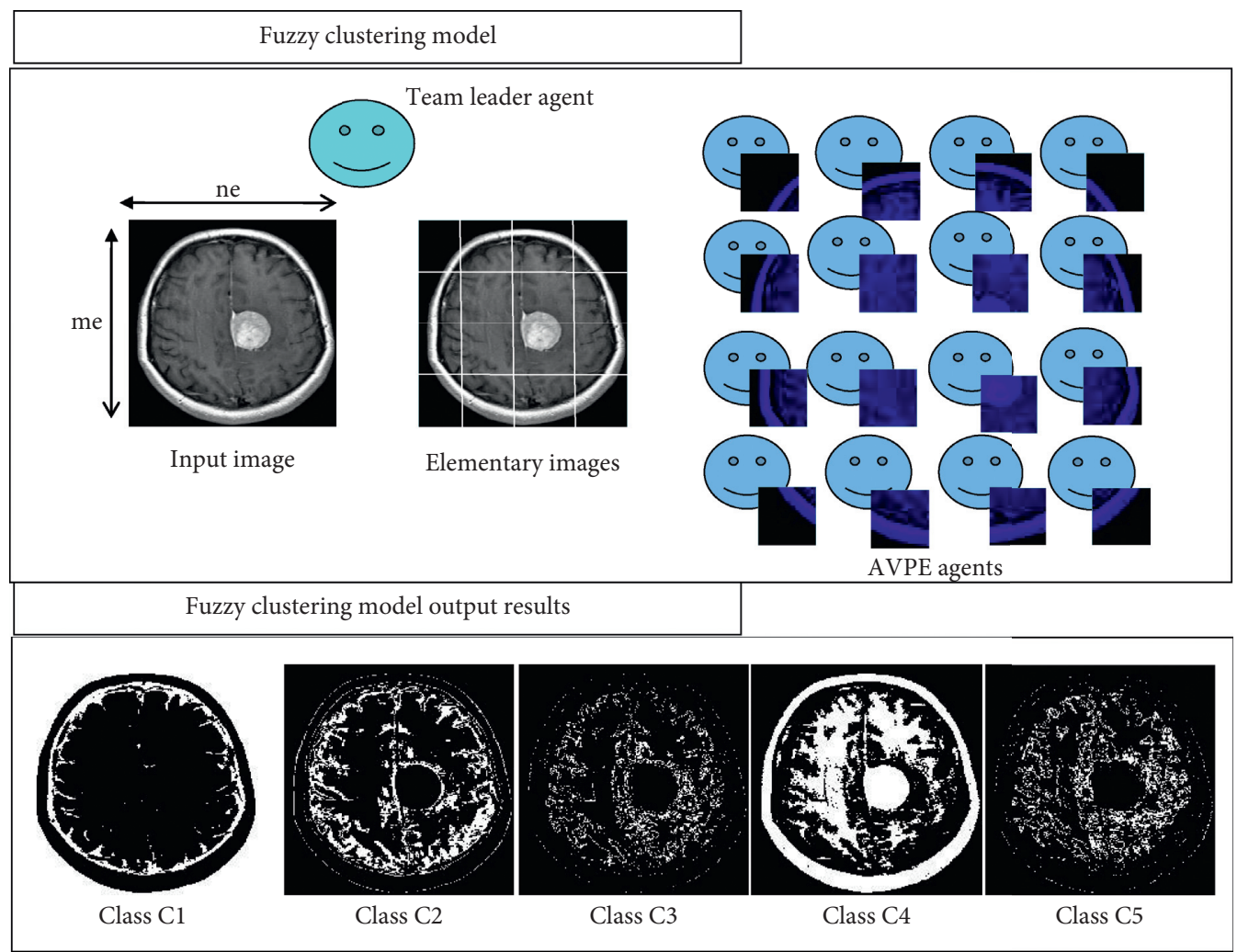

FIGURE 5: The proposed method's output clustering results.

TABLE 1: Different states of the DT2FL algorithm for image clustering starting from class centers initializations.

\begin{tabular}{lccccccccccc}
\hline \multirow{2}{*}{ Case } & \multicolumn{4}{c}{ Initial class centers } & \multicolumn{4}{c}{ Final class centers } & \multicolumn{2}{c}{ Number of iteration } \\
& C1 & C2 & C3 & C4 & C5 & C1 & C2 & C3 & C4 & C5 & \\
\hline Case 1 & 1.5 & 2.2 & 3.8 & 5.2 & 8.6 & 27.711 & 82.848 & 89.817 & 103.896 & 94.371 & 23 \\
Case 2 & 140.5 & 149.5 & 150.5 & 220.5 & 250.5 & 27.703 & 82.955 & 89.623 & 103.884 & 94.263 & 49 \\
\hline
\end{tabular}

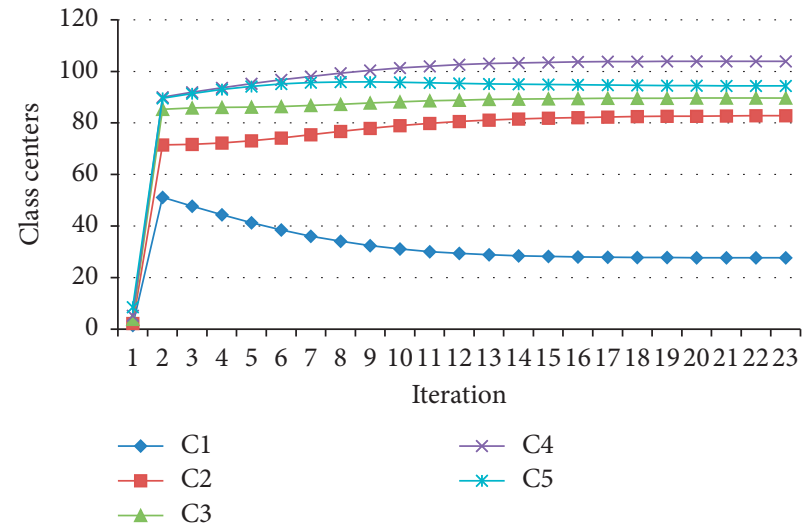

(a)

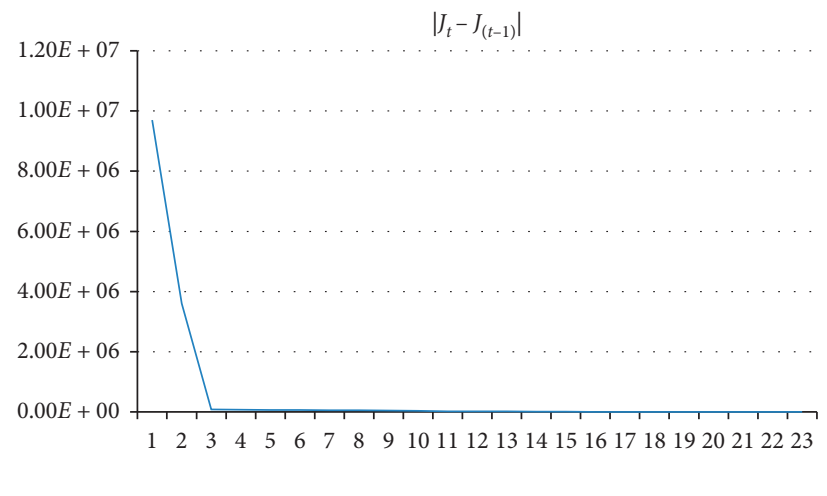

(b)

Figure 6: Dynamic convergence of class centers starting from class centers (c1, c2, c3, c4, c5) = $(1.5,2.2,3.8,5.2,8.6)$. (a) Class centers and (b) error. 


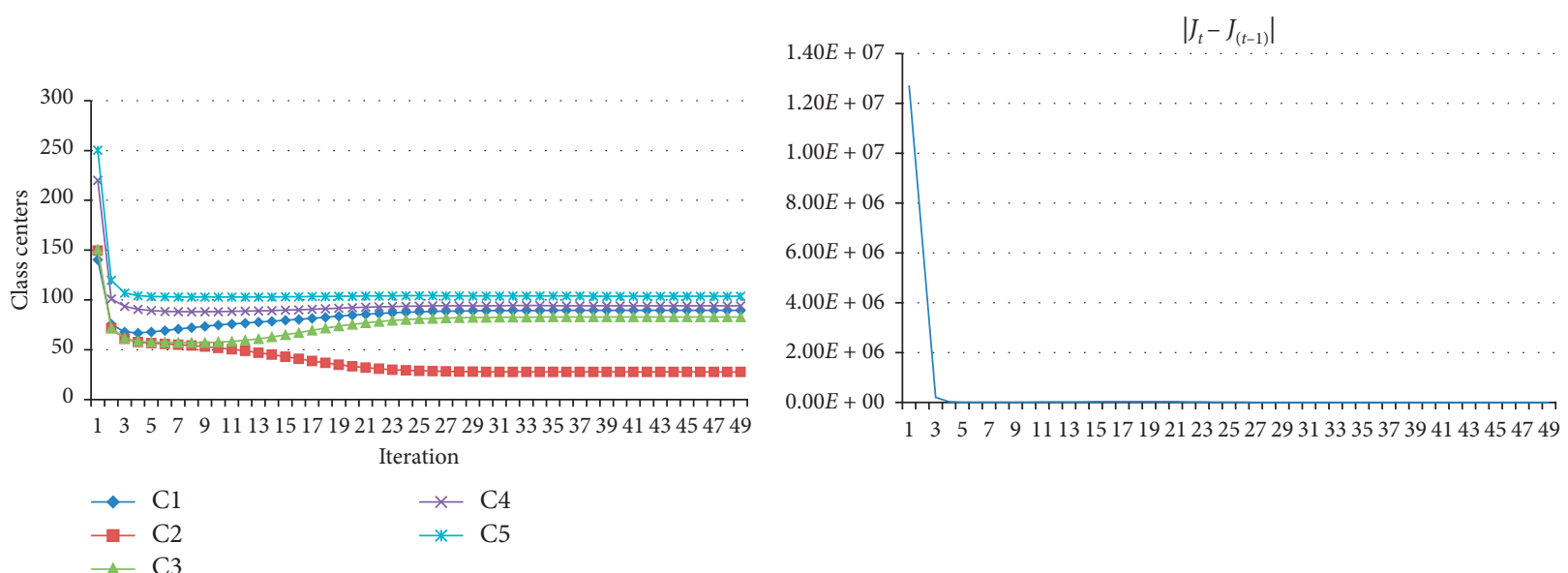

(a)

(b)

Figure 7: Dynamic convergence of class centers starting from class centers (c1, c2, c3, c4, c5) $=(140.5,149.5,150.5,220.2,250.5)$. (a) Class centers and (b) error.

TABLE 2: Comparing metric classification time of standard methods and distributed methods.

\begin{tabular}{|c|c|c|c|c|}
\hline \multicolumn{2}{|c|}{ Standard methods } & \multicolumn{3}{|c|}{ Distributed methods } \\
\hline $\begin{array}{l}\text { FCM classification Time } \\
(\mathrm{ms})\end{array}$ & $\begin{array}{l}\text { T2FCM classification Time } \\
(\mathrm{ms})\end{array}$ & $\begin{array}{l}\text { Number of } \\
\text { agents }\end{array}$ & $\begin{array}{l}\text { DFCM classification time } \\
(\mathrm{ms})\end{array}$ & $\begin{array}{l}\text { DT2FCM classification time } \\
(\mathrm{ms})\end{array}$ \\
\hline 1509 & 4318 & 1 & 1509 & 4318 \\
\hline- & - & 2 & 860 & 2461 \\
\hline- & - & 4 & 516 & 1477 \\
\hline- & - & 8 & 371 & 1062 \\
\hline- & - & 16 & 278 & 796 \\
\hline- & - & 32 & 266 & 762 \\
\hline
\end{tabular}

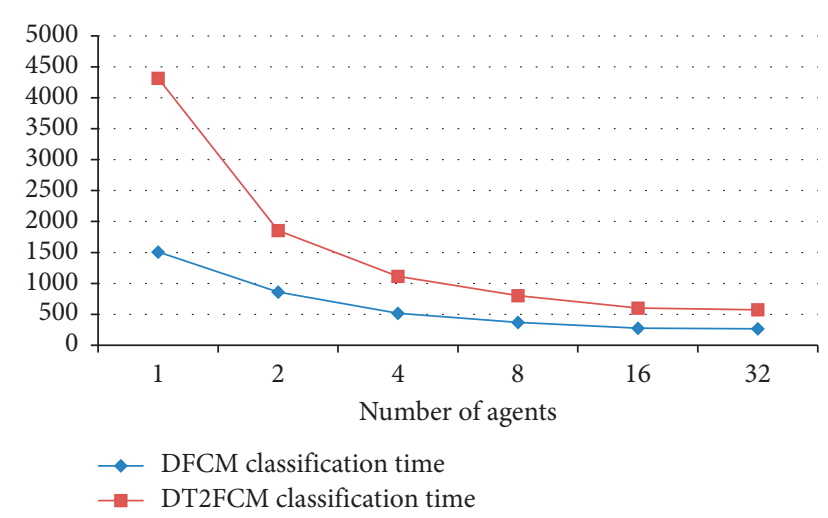

Figure 8: Classification time of the DT2FL and DFCM method based on the number of agents AVPE in the model.

Autonomous and hybrid Fuzzy logic system with two combined models: HPC computational intelligent medical data analysis, and cognitive decision making. The cognitive aspect of the method, combined with HPC clustering requirements provides a powerful system for medical applications.

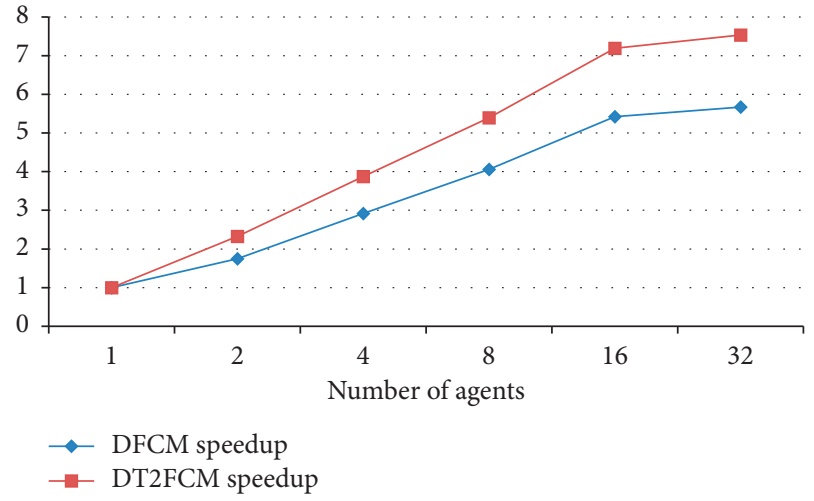

FIgURE 9: Speedup of DT2FL and DFCM methods based on the number of AVPE agents in the model.

\section{Conclusions}

In this paper, we presented a distributed type-2 Fuzzy logic method and its application for MRI medical image analysis. This method is implemented on machine learning modelbased mobile agents. The obtained results demonstrate the accuracy and efficiency of the proposed method. That is, it 
can meet the data requirements of scientists and engineers for testing and implementing their models. Indeed, the DT2FL method provides an aspect of adaptability with machine learning models. Thus, it allows reducing the complexity of the fuzzy clustering algorithms and modeling flexible and high performance systems for medical applications. Furthermore, intensive works are driven for designing an expert system for medical healthcare applications based on the proposed method within the deep learning technique and its implementation in cloud computing.

\section{Data Availability}

The data used to support the findings of this study are available from the corresponding author upon request.

\section{Conflicts of Interest}

The authors declare that there are no conflicts of interest regarding the publication of this paper.

\section{References}

[1] S. Rahimi, M. Zargham, A. Thakre, and D. Chhillar, "A parallel Fuzzy C-Mean algorithm for image segmentation," in Fuzzy Information," in Proceeedings of the IEEE Annual Meeting of the Fuzzy Information, 2004. Processing NAFIPS '04, vol. 1, pp. 234-237, Banff, Alberta, Canada, June 2004.

[2] T. Kwok, K. Smith, S. Lozano, and D. Taniar, Parallel Fuzzy C-Means Clustering for Large Data Sets BT-Euro-Par 2002 Parallel Processing, Springer, Berlin, Germany, 2002.

[3] M. A. Melgarejo and C. A. Peña-Reyes, "Hardware architecture and FPGA implementation of a type-2 fuzzy system," in Proceedings of the 14th ACM Great Lakes Symposium on VLSI, pp. 458-461, Boston, MA, USA, 2004.

[4] S. G. Andreas and C. Müller, Introduction to Machine Learning with Python: A Guide for Data Scientists, O'Reilly Media, Sebastopol, CA, USA, 1st edition, 2016.

[5] R. Sebastian and M. Vahid, Python Machine Learning: Machine Learning and Deep Learning with Python, Scikit-Learn, and TensorFlow, Packt, Birmingham, UK, 2nd edition, 2017.

[6] F. Bellifemine, G. Caire, and D. Greenwood, Developing Multi-Agent Systems with JADE, Wiley, West Sussex, UK, 2007.

[7] D. Isern, A. Moreno, D. Sánchez, Á. Hajnal, G. Pedone, and L. Z. Varga, "Agent-based execution of personalised home care treatments," Applied Intelligence, vol. 34, no. 2, pp. 155-180, 2011.

[8] A. Rodríguez-González, J. Torres-Niño, G. Hernández-Chan, E. Jiménez-Domingo, and J. M. Alvarez-Rodríguez, "Using agents to parallelize a medical reasoning system based on ontologies and description logics as an application case," Expert Systems with Applications, vol. 39, no. 18, pp. 1308513092, 2012.

[9] F. C. H. Rhee and C. Hwang, "A type-2 fuzzy C-means clustering algorithm," in Proceedings of the Joint 9th IFSA World Congress and 20th NAFIPS International Conference (Cat. No. 01TH8569), pp. 1926-1929, Vancouver, Canada, July 2001.

[10] F. Z. Benchara, M. Youssfi, O. Bouattane, and H. Ouajji, "A new scalable, distributed, fuzzy C-means algorithm-based mobile agents scheme for HPC: SPMD application," Computers, vol. 5, no. 3, 2016.
[11] R. John and S. Coupland, "Type-2 fuzzy logic: a historical view," IEEE Computational Intelligence Magazine, vol. 2, no. 1, pp. 57-62, 2007.

[12] O. Castillo and P. Melin, Type-2 Fuzzy Logic: Theory and Applications, Springer Publishing Company, Berlin, Germany, 1st edition, 2008.

[13] L. A. Zadeh, "From computing with numbers to computing with words. From manipulation of measurements to manipulation of perceptions," IEEE Transactions on Circuits and Systems I: Fundamental Theory and Applications, vol. 46, no. 1, pp. 105-119, 1999.

[14] R. John and S. Coupland, "Extensions to type-1 fuzzy logic: type-2 fuzzy logic and uncertainty," in In Computational Intelligence: Principles and Practice, D. B. F. G. Y. Yen, Ed., pp. 89-102, IEEE Computational Intelligence Society, 2006.

[15] M. Zarinbal, M. H. Fazel Zarandi, I. B. Turksen, and M. Izadi, "A type-2 fuzzy image processing expert system for diagnosing brain tumors," Journal of Medical Systems, vol. 39, no. 10, p. 110, 2015.

[16] O. M. D. S. A. Begum, "A rough type-2 fuzzy clustering algorithm for MR image segmentation," International Journal of Computer Applications, vol. 54, no. 4, pp. 4-10, 2012.

[17] T. Nguyen, A. Khosravi, D. Creighton, and S. Nahavandi, "Medical data classification using interval type-2 fuzzy logic system and wavelets," Applied Soft Computing, vol. 30, pp. 812-822, 2015.

[18] C. I. G, G. E. M. Oscar Castillo, and M. A. Sanchez, "Review of recent type-2 fuzzy image processing applications," Information, vol. 8, no. 3, 2017.

[19] P. Melin and O. Castillo, "A review on the applications of type-2 fuzzy logic in classification and pattern recognition," Expert Systems with Applications, vol. 40, no. 13, pp. 54135423, 2013.

[20] A. Mardani, R. E. Hooker, S. Ozkul et al., "Application of decision making and fuzzy sets theory to evaluate the healthcare and medical problems: a review of three decades of research with recent developments," Expert Systems with Applications, vol. 137, pp. 202-231, 2019.

[21] T.-Y. Chen, C.-H. Chang, and J.-f. Rachel Lu, "The extended QUALIFLEX method for multiple criteria decision analysis based on interval type-2 fuzzy sets and applications to medical decision making," European Journal of Operational Research, vol. 226, no. 3, pp. 615-625, 2013.

[22] E. Ramirez, P. Melin, and G. Prado-Arechiga, "Hybrid model based on neural networks, type-1 and type-2 fuzzy systems for 2-lead cardiac arrhythmia classification," Expert Systems with Applications, vol. 126, pp. 295-307, 2019.

[23] A. Kousar, N. Mittal, and P. Singh, “An improved hierarchical clustering method for mobile wireless sensor network using type-2 fuzzy logic BT," Proceedings of ICETIT 2019, pp. 128-140, Springer, Cham, Switzerland, 2020.

[24] J. Zhao, Y. Liu, L. Wang, and D. Wang, "A generalized heterogeneous type-2 fuzzy classifier and its industrial application," IEEE Transactions on Fuzzy Systems, p. 1, 2019.

[25] M. Dirik, O. Castillo, and A. F. Kocamaz, "Gaze-guided control of an autonomous mobile robot using type-2 fuzzy logic," Applied System Innovation, vol. 2, no. 2, 2019.

[26] F. Cuevas, O. Castillo, and P Cortes, Edited by O. Castillo, Ed., "Towards a control strategy based on type-2 fuzzy logic for an autonomous mobile robot," in Hybrid Intelligent Systems in Control, Pattern Recognition and Medicine, Studies in Computational Intelligence, P. Melin, Ed., Vol. 827, Springer, Cham, Switzerland, 2020. 
[27] A. Gkiokas and A. I. Cristea, "Cognitive agents and machine learning by example: representation with conceptual graphs," Computational Intelligence, vol. 34-2, pp. 603-634, 2018.

[28] M. Francisco, Y. Mezquita, S. Revollar, P. Vega, and J. F. De Paz, "Multi-agent distributed model predictive control with fuzzy negotiation," Expert Systems with Applications, vol. 129, pp. 68-83, 2019.

[29] J. Ropero, A. Gómez, A. Carrasco, and C. León, “A fuzzy logic intelligent agent for information extraction: introducing a new fuzzy logic-based term weighting scheme," Expert Systems with Applications, vol. 39, no. 4, pp. 4567-4581, 2012.

[30] B. Sabetghadam, F. Shabaninia, M. Vaziri, and S. Vadhava, "Type-2 fuzzy multiagent traffic signal control," in Proceedings of the 2012 IEEE 13th International Conference on Information Reuse \& Integration (IRI), pp. 509-512, Las Vegas, NV, USA, August 2012.

[31] K.-Y. Lu and C.-C. Sy, "A real-time decision-making of maintenance using fuzzy agent," Expert Systems with Applications, vol. 36, no. 2, pp. 2691-2698, 2009.

[32] P. G. Balaji, D. Srinivasan, and C. Tham, "Coordination in distributed multi-agent system using type-2 fuzzy decision systems," in Proceedings of the 2008 IEEE International Conference on Fuzzy Systems (IEEE World Congress on Computational Intelligence), pp. 2291-2298, Hong Kong, China, June 2008.

[33] Y. Jarraya, S. Bouaziz, H. Hagras, and A. M. Alimi, "A multiagent architecture for the design of hierarchical interval type2 beta fuzzy system," IEEE Transactions on Fuzzy Systems, vol. 27, no. 6, pp. 1174-1188, 2019. 